ARTICLE

Received 1 Oct 2012 | Accepted 21 Nov 2012 | Published 18 Dec 2012

DOl: 10.1038/ncomms2312 OPEN

\title{
A visible dominant marker for insect transgenesis
}

\author{
Mizuko Osanai-Futahashi ${ }^{1}$, Takahiro Ohde ${ }^{2}$, Junya Hirata ${ }^{2}$, Keiro Uchino ${ }^{1}$, Ryo Futahashi ${ }^{3}$, Toshiki Tamura ${ }^{1}$, \\ Teruyuki Niimi ${ }^{2} \&$ Hideki Sezutsu ${ }^{1}$
}

Transgenesis of most insects currently relies on fluorescence markers. Here we establish a transformation marker system causing phenotypes visible to the naked eye due to changes in the color of melanin pigments, which are widespread in animals. Ubiquitous overexpression of arylalkylamine- $\mathrm{N}$-acetyl transferase in the silkworm, Bombyx mori, changes the color of newly hatched first-instar larvae from black to a distinctive light brown color, and can be used as a molecular marker by directly connecting to baculovirus immediate early 1 gene promoter. Suppression of black pigmentation by Bm-arylalkylamine- $\mathrm{N}$-acetyl transferase can be observed throughout the larval stages and in adult animals. Alternatively, overexpression in another gene, B. mori $\beta$-alanyl-dopamine synthetase (Bm-ebony), changes the larval body color of older instars, although first-instar larvae had normal dark coloration. We further show that ectopic Bm-arylalkylamine- $\mathrm{N}$-acetyl transferase expression lightens coloration in ladybird beetle Harmonia axyridis and fruit fly Drosophila melanogaster, highlighting the potential usefulness of this marker for transgenesis in diverse insect taxa.

\footnotetext{
${ }^{1}$ Transgenic Silkworm Research Unit, National Institute of Agrobiological Sciences (NIAS), 1-2 Owashi, Tsukuba, Ibaraki 305-8634, Japan. ${ }^{2}$ Graduate Schoo of Bioagricultural Sciences, Nagoya University, Chikusa, Nagoya 464-8601, Japan. ${ }^{3}$ National Institute of Advanced Industrial Science and Technology (AIST), Tsukuba, Ibaraki 305-8566, Japan. Correspondence and requests for materials should be addressed to M.O.-F. (email: mfutahashi@nias.affrc.go.jp) or to H.S. (email: hsezutsu@affrc.go.jp).
} 
G enetic modification of insects is an essential and basic technique for studying developmental biology, as well as for applications such as pest control and recombinant protein production ${ }^{1}$. Transgenic techniques have been developed for many dipterans and lepidopteran insects ${ }^{2,3}$, and also for insects in other orders such as the red flour beetle Tribolium castaneum $^{4}$, the ladybird beetle Harmonia axyridis ${ }^{5}$, the sawfly Athalia rosae $e^{6}$ and the cricket Gryllus bimaculatus ${ }^{7}$.

In Drosophila melanogaster, transgenic flies are generally identified by mutant-based genetic markers with visible phenotypes (for example, the white gene for eye pigmentation) ${ }^{8}$. However, most insect species do not have a set of suitable recipient mutant strains and the functional transgene to rescue the phenotype. Instead, fluorescence markers such as 3xP3enhanced green fluorescent protein (EGFP), which drives the eyespecific expression of green fluorescent protein, are used ${ }^{9-12}$. As this transgene is dominant, it is especially useful for nontraditional model insects. However, screening under a fluorescence microscope is a laborious procedure for large-scale projects, and insect eyes normally have dark pigmentation, which makes detecting fluorescence difficult. Thus, the development of a dominant visible marker applicable to various strains would be beneficial for studies of non-Drosophila insects.

For transgenic screening, it would be convenient if the altered phenotype could be easily identified externally at an early developmental stage. Suitable characteristics include unique pigmentation of eggs or neonatal larvae. One of the major pigments in insects is melanin, which is usually black or brown in color and is widespread in the exoskeletal cuticle ${ }^{13-19}$. Melaninrelated pigments are mainly derived from dopamine ${ }^{18,20,21}$, which is synthesized from tyrosine by the enzymes tyrosine hydroxylase and dopa decarboxylase.

Besides black and brown melanin pigments, dopamine is involved in the synthesis of light-coloured pigments and also in sclerotization. $N$ - $\beta$-alanyl-dopamine (NBAD), a derivative of dopamine, is a precursor for the yellow/red cuticular pigments of Drosophila $^{18}$ and the larva of swallowtail butterfly, Papilio $x_{\text {uthus }}{ }^{15}$, and for NBAD-o-quinone, which crosslinks cuticle proteins in sclerotization ${ }^{22,23}$. Another derivative of dopamine, $\mathrm{N}$-acetyl-dopamine (NADA), is a precursor for NADA-oquinone, which is also involved in sclerotization of transparent or very light-coloured cuticles ${ }^{22-24}$. Dopamine is converted to NBAD by the $\beta$-alanyl-dopamine synthetase encoded by ebony gene, whereas NADA is synthesized from dopamine by arylalkylamine- $N$-acetyl transferase (aaNAT) activity.

The ebony mutant of Drosophila has darker overall coloration than wild type, and the ectopic expression of Dm-ebony changes the dark coloration of the adult abdomen to yellow ${ }^{18}$. In the silkworm Bombyx mori, the mutant allele of Bm-ebony is called sooty, which displays a distinct black pupal coloration instead of the normal light brown color ${ }^{16}$. The larvae and adults of sooty have a smoky color, which is less conspicuous than in pupae.

The identity of the gene responsible for aaNAT activity in cuticle sclerotization is less clear. In insects, aaNAT activity is not only involved in sclerotization, but also in neurotransmitter catabolism and melatonin precursor formation $25-32$. Two aaNAT genes have been identified in Drosophila ${ }^{25,26}$, one of which is expressed in the brain. However, their involvement in sclerotization has not been reported, leaving open the possibility that an unidentified aaNAT enzyme may be involved in sclerotization in this insect. From the recent results of in vitro aaNAT activity analysis, aaNAT1 and aaNAT2 are assumed to be involved in sclerotization, and neurotransmitter inactivation in Aedes aegypti ${ }^{33}$. In B. mori, only one aaNAT gene, Bm-aaNAT has been characterized ${ }^{28}$. Notably, the mutant allele melanism $(\mathrm{mln})$ does not display serious defects in sclerotization, but the adult shows darker overall pigmentation, and larvae show dark pigmentation in the head, forelegs and tail spot ${ }^{34,35}$. However, no reports exist for phenotypic effects of the mln mutant in the pupa and most parts of the larval epidermis ${ }^{34,35}$. Several aaNAT-like genes have been predicted in the B. mori genome based on sequence similarity ${ }^{35}$, which suggests that some of them may function in sclerotization in different regions and stages. As the substrates of aaNAT include dopamine ${ }^{27,31}$, it may be able to interfere with the synthesis of the dark dopamine-melanin. However, ectopic expression of aaNAT has never been tested in vivo in insects for converting dopamine to NADA.

In silkworms, the neonatal larvae are black in color due to melanin, and are traditionally called 'ants' in Japanese. The development of a transgene that could change the color of the cuticle in insects of this stage in a dominant manner would be beneficial for the production and maintenance of transgenic silkworm strains.

In this study, we tested whether ectopic expression of $B m$-aaNAT and Bm-ebony interferes with the black melanin pigmentation of silkworms. Using the GAL4/UAS binary system, we found that ectopic expression of the Bm-aaNAT gene alters the coloration of neonatal larva from a dark ant-like color to light brown. In addition, overexpression of the Bm-aaNAT and Bmebony genes changed the pigmentation in the cuticle of second- to final-instar larva and the adult antennae from black/brown to a lighter color. We also demonstrate that expressing the $B m$-aaNAT gene under the direct control of a constitutive baculovirus immediate early 1 (IE1) gene promoter produced a color transformation in neonatal larvae. Furthermore, the $B m$-aaNAT gene had ability to lighten dark melanin coloration in ladybird beetle $H$. axyridis and fruit fly $D$. melanogaster, which strongly indicates the utility of Bm-aaNAT gene as a dominant molecular marker in insects.

\section{Results}

Bm-aaNAT lightens body color of neonatal larvae in silkworm. To express Bm-aaNAT and Bm-ebony in B. mori, we first used the binary GAL4/ upstream activating sequence (UAS) system. Effector vectors that carried the Bm-aaNAT gene or Bm-ebony gene linked to an UAS and an EGFP reporter gene under the control of the eye-specific $3 \mathrm{xP} 3$ promoter were constructed (Fig. 1a). The effector lines were generated by germline transformation. After sib mating and selection of G1 progeny based on the presence of EGFP fluorescence, G1 male moths were crossed with female moths of the actin A3-GAL4 driver line 193-2, which drives ubiquitous expression of GAL4, and can be identified by the 3xP3-DsRed2 marker (Fig. 1a) ${ }^{36}$. The overexpression of $B m$-aaNAT and Bm-ebony was first tested in the w1-pnd strain, which is generally used to produce transgenic silkworms because the white-egg phenotype is convenient for fluorescence screening of the embryonic eye. In contrast to the blackish coloration of control $w 1$ neonatal larvae, the head and the body of the newly hatched larvae were light brown in color in all three actin A3GAL4; UAS-NAT lines (Fig. 1b, Supplementary Fig. S1 online). This suggests that Bm-aaNAT interfered with the dopaminemelanin synthesis cascade in the cuticle of neonatal larvae, and produced NADA, leading to lighter melanin pigmentation. However, only a subtle change in coloration was observed in actin A3-GAL4; UAS-ebony neonatal larvae (Fig. 1c, Supplementary Fig. S1), although we checked several lines.

$B m-a a N A T$ and Bm-ebony suppress larval black pigmentation. In wild-type silkworm strains, after the second instar, the epidermis is mainly white and opaque due to the accumulation of uric acid granules, and has several black markings consisting of 
melanin and ommochrome pigmentation. To observe the effects of ubiquitous overexpression of Bm-aaNAT and Bm-ebony in older instar larvae, we chose to use the black striped strain striped $\left(p^{S}\right.$; Fig. 2). This strain has a wide black stripe in each segment induced by the dominant striped gene $p^{S}$, in addition to the melanin pigmentation in eyespot markings of the second thoracic segment, crescent markings in the second abdominal segment, and star spot markings in the fifth abdominal segment, which are present in wild-type strains. We crossed the striped gene $p^{S}$ homozygotes with actin A3-GAL4; UAS-NAT and actin A3GAL4; UAS-ebony lines (Supplementary Fig. S2). As expected, the ectopic expression of aaNAT caused marked changes in pigmentation although the coloration of each instar differed. In the both second- and the third-instar larvae of actin A3-GAL4; UASNAT $\left(p^{S}\right)$ lines, the black body stripes, and the crescent and star spot markings on the second and fifth abdominal segments were a very light tan color (Supplementary Fig. S3). In the fourth-instar larvae of these lines, the black body stripes were a light brownish grey color, and crescent markings and star spot markings were a lighter brown color. In addition, the brown-coloured area was larger compared with wild type, especially in the crescent markings (Supplementary Fig. S4). In the fifth instar, the stripes were light grey instead of black (Fig. 2). Notably, the pigmented areas of eyespot markings in the second thoracic segment, crescent markings, and star spot markings were markedly smaller and the shapes of the crescent and star spot markings were altered (Fig. 2). These observations indicate that the ectopic expression of $B m$-aaNAT not only affects the synthesis of melanin pigments in neonatal larvae, but throughout the larval stages.

Although the actin A3-GAL4; UAS-ebony showed no marked changes in neonatal larval coloration, a change in pigmentation was observed from the second instar in $p^{S}$-crossed individuals. Like actin A3-GAL4; UAS-NAT $\left(p^{S}\right)$ lines, larval pigmentation differed between stages, but was also different from aaNAToverexpressing lines. The color of the markings in the secondinstar larvae of actin A3-GAL4; UAS-ebony $\left(p^{S}\right)$ lines changed from black to pale yellow. In the third-instar larvae, most of the black regions changed to a darker orange-yellow color, which was a

UAS-NAT/ 3xP3EGFP

UAS-ebony/ 3xP3EGFP

actin A3-GAL4/ 3xP3DsRed2

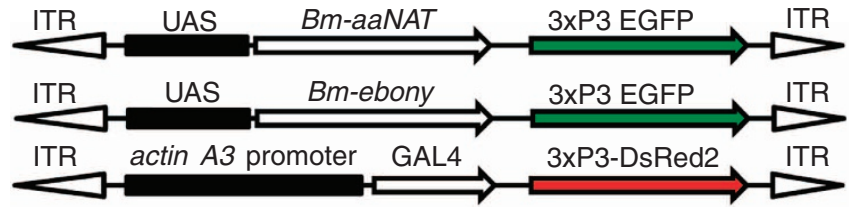

b

actin A3-GAL4; UAS-NAT

actin A3-GAL4 (control)

C

actin A3-GAL4; UAS-ebony

UAS-ebony (control)
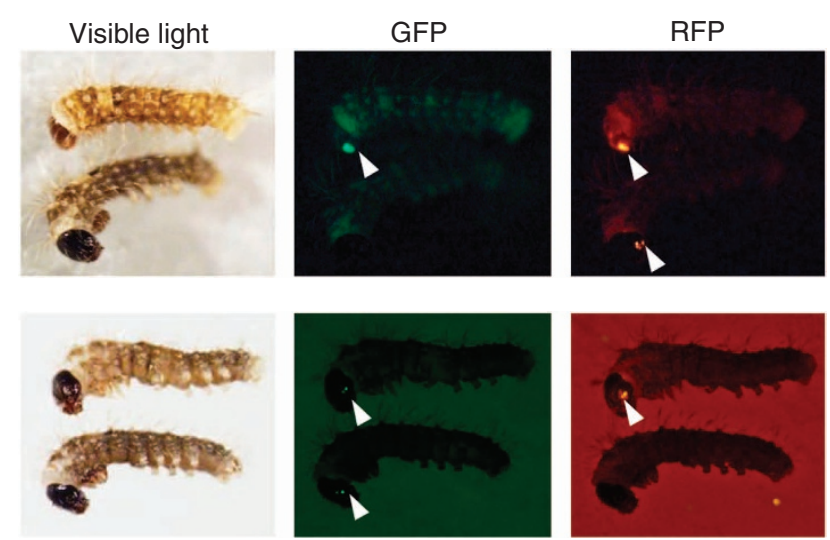

Figure 1 | Bm-aaNAT overexpression alters cuticle coloration in neonatal silkworm larvae. (a) Structures of UAS-NAT/3xP3EGFP, UAS-ebony/3xP3EGFP and actin A3-GAL4/3xP3DsRed2 (not to scale). 3xP3, eye-specific promoter; ITR, inverted terminal repeat of the piggyBac transposon. Effect of ectopic expression of Bm-aaNAT (b) and Bm-ebony (c) using the GAL4/UAS binary system (w1-pnd background). Left, bright field; middle, GFP; and right, red fluorescent protein (RFP). White arrowheads indicate fluorescence in the ommatidia.
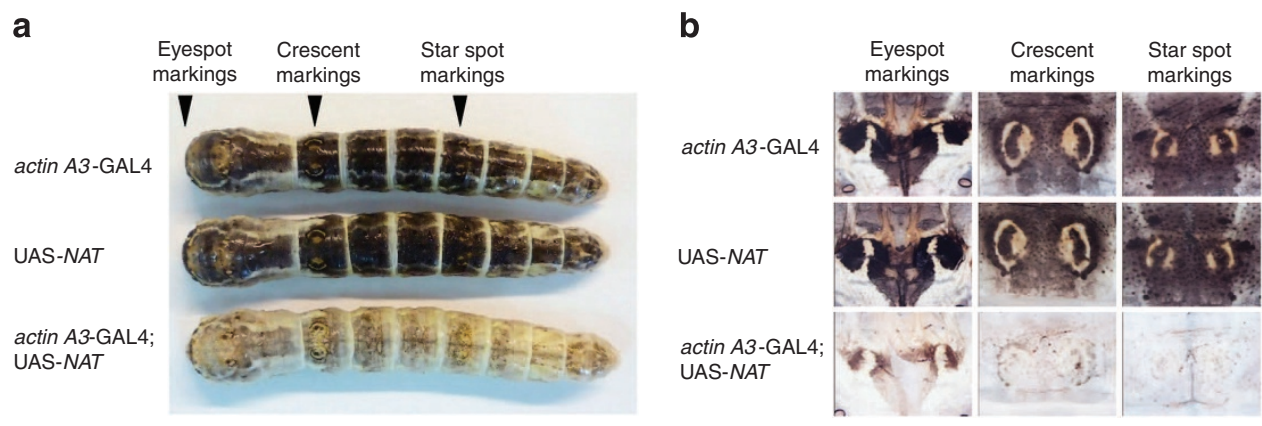

Figure 2 | Bm-aaNAT overexpression lightens pigmentation in the black striped silkworm strain. The fifth-instar larvae shown here have the dominant striped $\left(p^{S}\right)$ genetic background. (a) Dorsal view of the larvae. The positions of the markings magnified in $\mathbf{b}$ are indicated by the arrowheads. (b) Magnified images of the larval markings. 
different from that in actin A3-GAL4; UAS-NAT $\left(p^{S}\right)$ larvae of the same instar (Fig. 3). Although black pigmentation was not observed in the third-instar larvae of actin A3-GAL4; UAS-NAT $\left(p^{S}\right)$ lines, it remained in actin A3-GAL4; UAS-ebony $\left(p^{S}\right)$ lines in several areas such as the margins of the crescent marking. In the fifth-instar larvae, ectopic ebony overexpression did not induce yellowish coloration, and the change of pigmentation was modest compared with that in younger larvae. However, they could be distinguished from non-overexpressing lines by the loss of pigmentation in the lateral area especially in areas surrounding the spiracles, and in the dorsal region of the second and third thoracic segments (Supplementary Fig. S5).

As the silkworm tracheae are also pigmented with black melanin, we inspected the tracheae of 3-day-old fifth-instar larvae of actin A3-GAL4; UAS-NAT and actin A3-GAL4; UAS-ebony lines, and found them to be pale brown in color, indicating that dopamine was converted into NADA or NBAD instead of dopamine-melanin (Supplementary Fig. S6 online). Taken together, our data show that ectopic expression of Bm-aaNAT affected melanization of the epidermal cuticle in larvae of all stages and the tracheae, and that ectopic expression of Bm-ebony affected the melanization of the second- to fifth-instar larvae and the tracheae.

Bm-aaNAT and Bm-ebony lighten adult antenna coloration. No clear differences were observed between control insects and actin A3-GAL4; UAS-NAT- and actin A3-GAL4; UAS-ebonycarrying insects in the wing scales and body hair of adult silk moths, which are normally white. However, a distinct change in the color of the antennae was observed (Fig. 4). In contrast to the normal brown pigmentation, the antennae of Bm-aaNAT- and $B m$-ebony-overexpressing insects were light brown in color. This suggests that the pigment of the antennae in adult silk moths is derived from dopamine-melanin, which was converted into NADA and NBAD by ectopically expressed Bm-aaNAT and Bm-ebony, respectively.

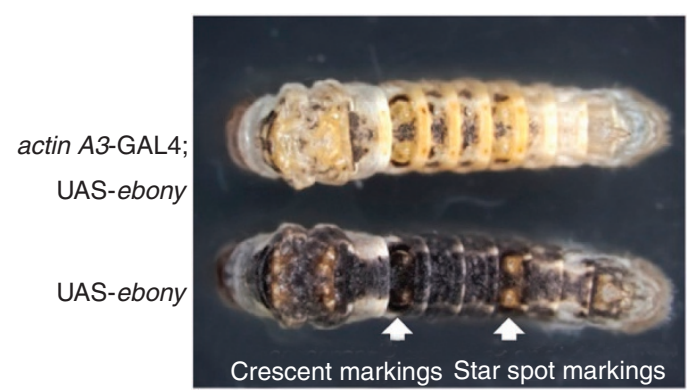

Figure 3 | Bm-ebony overexpression alters silkworm larval pigmentation in older instars. The black striped silkworm $\left(p^{S}\right)$ strain, which the effect on melanin pigmentation is easy to observe is used. The dorsal view of thirdinstar larvae is shown. Ubiquitous overexpression of Bm-ebony changed most areas of black pigmentation.
Development of a dominant marker with the Bm-aaNAT gene. As the phenotype produced by ectopic Bm-aaNAT expression seemed to be ideal for a transformation marker, we next tested whether Bm-aaNAT directly linked to a promoter alters the black melanin coloration. We tested the $B$. mori actin $A 3$ promoter and baculovirus IE1 gene promoter to drive expression of the transgene (Fig. 5a). Although no large difference in coloration was observed using the actin A3 promoter, the body color of neonatal larvae carrying the IE1-NAT transgene was light brown (Fig. 5b, left panel, white arrowheads, Supplementary Fig. S7) in all eight G1 strains expressing EGFP fluorescence in the eye, and the difference in color was easily distinguishable without the aid of a microscope. The transformation efficiency for IE1-NAT transgene was $19 \%(8 / 43)$, which compared favourably with that achieved with the actin A3-EGFP transgene (14\%) and 3xP3EGFP transgene $(12 \%)$ using the same system ${ }^{37}$. In addition, no significant difference was detected in the survival rate, and developmental time was only delayed for average of 1 day in IE1-NAT G1 silkworms (average $81.3 \%$, s.d. $=15.1 \%, n=4$ ) compared with their non-transgenic siblings (average $87.4 \%$, s.d. $=3.9 \%, n=4$; $P=0.47$, Student's $t$-test), and the subesequent generation also grew normally, and had no notable difference with non-transgenic siblings in fecundity (Supplementary Fig. S8, Supplementary Table $\mathrm{S1}$ ). Furthermore, the light brown coloration was observed for larvae with $50 \%$ segregation obtained from an F1 backcross, which suggests that insertion of a single copy is enough to alter pigmentation. Moreover, when we used C146 (a commercial silkworm strain homozygous for the wild-type w1 (kynurenine 3mono oxygenase) gene, which has brown ommochrome pigments in the epidermis in addition to melanin pigments; and 1.9-fold cocoon yield of w1-pnd strain, Supplementary Fig. S8), as the host for germline transformation, G1 transformants could easily be distinguished without microscopy by their orange-brown color (Fig. 5b, right panel, white arrowheads), with only small decrease in cocoon weight (Supplementary Fig. S8), verifying that the IE1-NAT transgene can function as a dominant visible transgenic marker in practical wild-type silkworm strains.

Bm-aaNAT suppresses pigmentation also in other insect taxa. Finally, to check the potential usefulness of $B m-a a N A T$ in diverse insect taxa, we tested heterologous expression of $B m$-aaNAT in fruit fly $D$. melanogaster and ladybird beetle $H$. axyridis. First, we generated UAS-NAT effector fly lines with the same construct that used for silkworm, and crossed with heat shock protein 70 (hsp)-GAL4, and pannier-GAL4 (ref. 38) (which expresses GAL4 in a broad stripe along the dorsal midline of developing flies) lines. As a result, hsp-GAL4; UAS-NAT individuals had overall clearly lighter pigmentation and white bristles in the thoracic segment (Fig. 6a), and black pigmentation of pannier-GAL4; UAS-NAT individuals was diminished in the pannier expression domain (Fig. 6b, bracket), in a similar manner to the pannierGAL4; UAS-ebony phenotype reported by (ref.12). The light coloration phenotype was observed for both of the two UAS-NAT
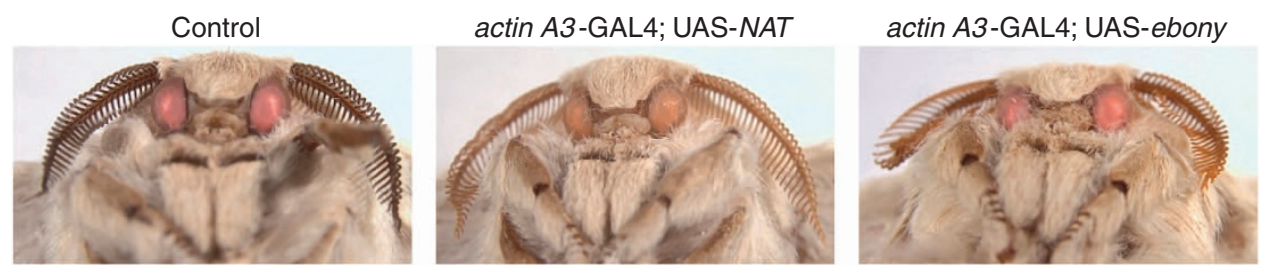

Figure 4 | Ectopic expression of Bm-aaNAT and Bm-ebony lightens adult antenna coloration. Moths carrying only actin A3-GAL4/3xP3DsRed2 were used as controls. 
a IE1-NAT/ 3XP3EGFP

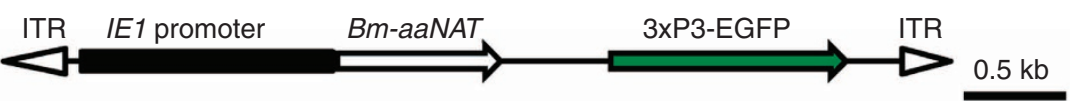

b
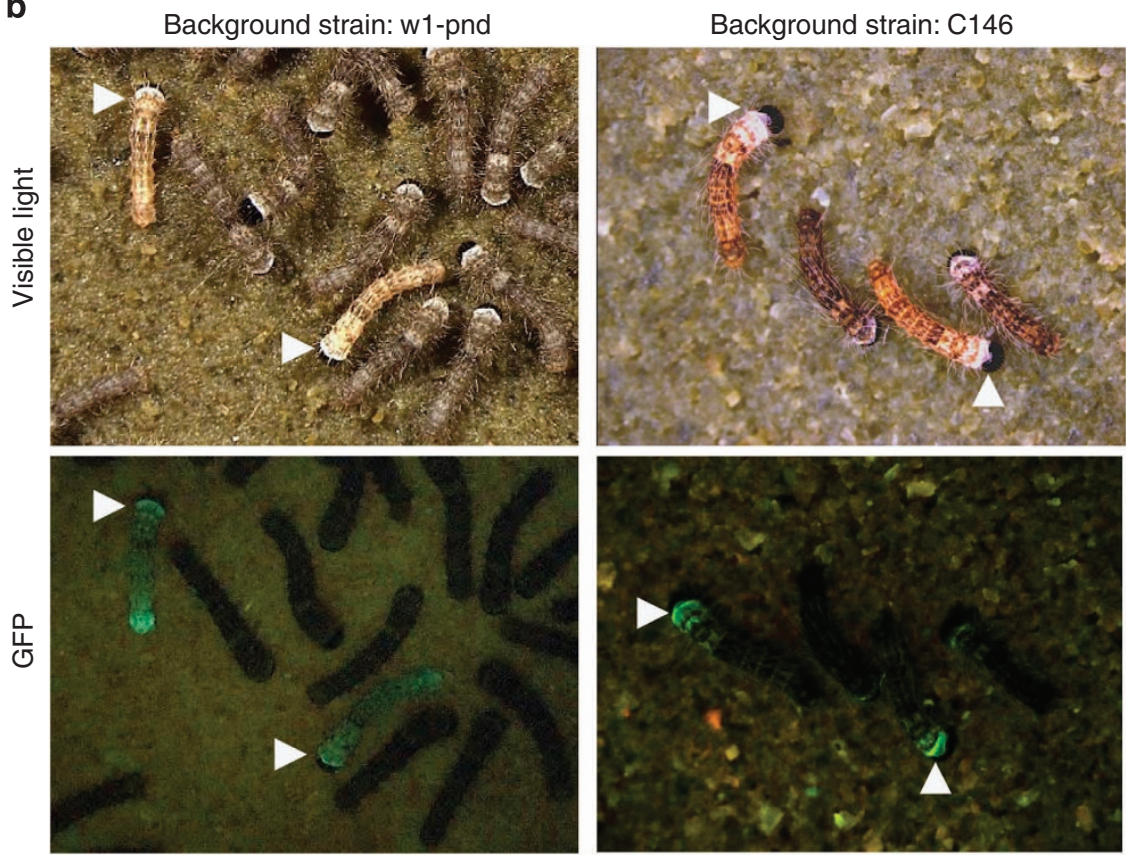

Figure 5 | Development of a dominant marker IE1-NAT that alters neonatal larval coloration. (a) Structure of the IE1-NAT/3xP3EGFP transgene. Bm-aaNAT expression was under the direct control of the constitutive baculovirus IE1 promoter. (b) Neonate larvae carrying IET-NAT transgene can be easily distinguished by its light coloration (white arrowheads), whether w1-pnd (kynurenine 3-mono oxygenase (KMO)-deficient strain, left panels) or commercial strain C146 (with intact KMO gene, right panels) is used as the host strain for transgenesis. Top panels, bright field; bottom panels, GFP filter.

effector fly lines we obtained. These results indicate that the ectopic expression of Bm-aaNAT suppress black coloration also in Drosophila. For the ladybird beetle $H$. axyridis, we used the IE1 promoter and the Drosophila hsp promoter (Supplementary Fig. S9A) to drive Bm-aaNAT expression (Fig. 6c). Although the IE1-NAT transgene did not show any coloration effects, one of the three transgenic strains with $h s p-N A T$ transgene produced markedly light-coloured larvae (Fig. 6c, Supplementary Fig. S9B), possibly due to the influence of a nearby enhancer. The light coloration phenotype of this strain was enhanced by heat shock treatment (Fig. 6c), and Bm-aaNAT expression was confirmed by reverse transcription-polymerase chain reaction (Supplementary Fig. S9C), supporting that the light coloration is caused by the effect of Bm-aaNAT. Overall, these results indicate that $\mathrm{Bm}$ aaNAT has the ability to lighten melanin coloration also in several insect taxa other than Lepidoptera.

\section{Discussion}

We have shown that ectopic expression of Bm-aaNAT or Bmebony is sufficient to alter the black melanin pigmentation, which demonstrates their potential usefulness as dominant transformation markers. Although ubiquitous expression of Bm-aaNAT or Bm-ebony may affect the dopamine amount in the nervous system, there were no serious effects on fitness (Supplementary Fig. S8) and behaviour such as eating and mating in the present experiments. In addition to facilitating the screening procedure, it is also beneficial regarding the choice of host strains for germline transformation. For instance, in silkworms, $w 1$ mutant strain with white egg and eyes have been the feasible host for germline transformation because the eye-specific fluorescent markers are difficult to detect in wild-type strains with dark eggs and eyes. This has been a drawback for the analysis of most silkworm strains other than $w 1$, and it is preferable to use commercial strains for production of biomaterials such as spider silk ${ }^{39}$ because of their high productivity of silk. The use of aaNAT marker would be of benefit on such cases, and moreover, the widespread distribution of melanin-related pigments, conservation of the melanin synthesis pathway and ability of aaNAT to suppress black coloration even in flies and ladybird beetles (Fig. 6, Supplementary Fig. S9) suggest its potential as a transformation marker in diverse insect taxa. In the ladybird beetle, suppression of black pigmentation occurred only in the larval stage of a single strain out of three transgenic strains and was not observed in the adult stage, suggesting that the $B m$-aaNAT expression in this strain is under the influence of a nearby enhancer. However, as the IE1 promoter is derived from Autographa californica polyhedrosis virus, it is likely that IE1NAT transgene could be directly applied to lepidopteran insects, which many species are known to be agricultural pests, and practical use of Bm-aaNAT gene should be possible for other taxa in combination with suitable promoters. Furthermore, aaNAT marker can be used in parallel with fluorescent markers because it does not interfere with the fluorescent signals, which enables the addition of an extra transgene.

The data presented in this study also have significant impact for the understanding of molecular basis of melanin/cuticle synthesis. Although NADA is the most common precursor for colourless cuticle in the melanin/cuticle synthesis pathway of insects $^{22,23}$, the gene involved in its synthesis had long remained 
a

UAS-NAT (control) hsp-GAL4;UAS-NAT
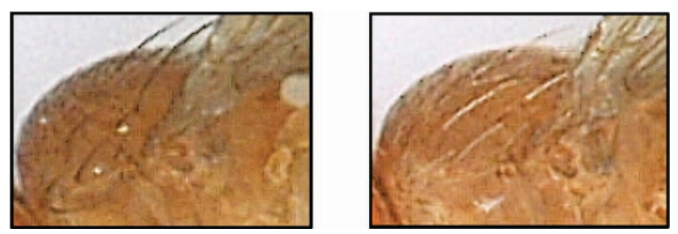

b

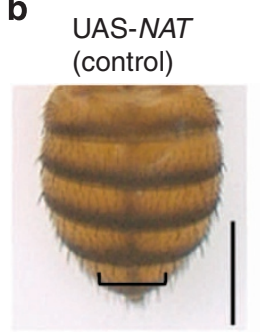

C

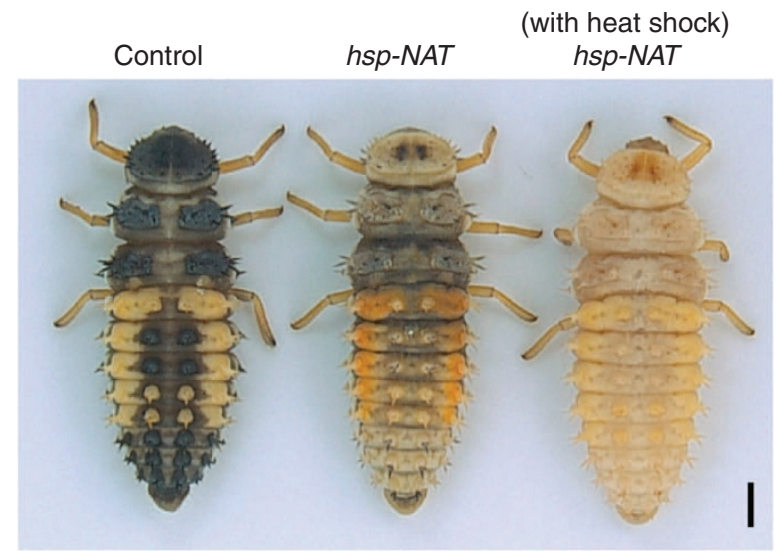

Figure 6 | Bm-aaNAT lightens coloration in other insect orders.

$(\mathbf{a}, \mathbf{b})$ Effect of ectopic Bm-aaNAT expression in D. melanogaster. The flies are at least 4 days $(100 \mathrm{~h}$ ) old. Scale bar, $1 \mathrm{~mm}$ (a), $0.5 \mathrm{~mm}$ (b). (a) Shows the effect of Bm-aaNAT driven by hsp-GAL4. The fly shows overall lighter pigmentation, and as shown in the bottom panels (the magnified view of the square in the top panel) bristle coloration are also suppressed by $B m$-aaNAT overexpression. (b) Shows the effect of Bm-aaNAT driven by pannier-GAL4, which drives expression in a broad stripe along the dorsal midline (brackets). Abdomen of adult flies is shown. (c) Effect of hsp-NAT transgene on $\mathrm{H}$. axyridis fourth-instar larvae. Left, insect with no transgene; middle, insect with hsp-NAT transgene; right, insect with hsp-NAT transgene with heat shock treatment. Scale bar, $1 \mathrm{~mm}$.

unclear, until the recent discovery that Bm-aaNAT gene was disrupted in the B. mori mutant melanism ${ }^{34,35}$. Our results provide a direct evidence that $\mathrm{Bm}$-aaNAT has the ability to lighten coloration in adults as well as the whole epidermal cuticle in silkworm larvae. Although Bm-aaNAT mutation in the mutant melanism has little effect on larval coloration, overexpression of $B m$-aaNAT in this study induced marked changes throughout the larval stages (Figs 1, 2, 4-6, Supplementary Figs S1, S3, S4 and S7 online). The aaNAT gene was conserved in other lepidopterans such as monarch butterfly Danaus plexippus (GenBank accession code EHJ67891.1), and also was detected in 23 species out of 25 arthropods (excepting tick Ixodes scapularis and jewel wasp Nasonia vitripennis) when searched by OrthoDB (http:// cegg.unige.ch/orthodb4). Recently, upregulated aaNAT expression linked with yellow pigmentation was reported in Heliconius butterfly hindwing ${ }^{40}$, which suggests that aaNAT functions as a modifier in melanin pigmentation of other insects.

As for ebony, it has been described as a suppressor of black coloration in Drosophila that delimits the black pigmentation on the wing and abdomen ${ }^{18,41}$, and reported to change black melanin pigmentation to a tan color by ectopic expression (Fig. 7$)^{18}$. Ebony is also reported to be associated with the yellow/ red pigments in the larval eyespot markings and adult wing of the swallowtail and Heliconius butterflies ${ }^{40,42-44}$. Our results showing that ectopic ebony expression alters the color of melanin pigments in larvae and adults are consistent with these reports, and the conservation of the ebony gene in arthropods (24 out of 25 species in OrthoDB, not detected only in I. scapularis) suggest that it may contribute to light-coloured pigmentation in other lepidopterans.

Notably, ectopic expression of Bm-aaNAT and Bm-ebony had different effects in silkworm larvae. In general, Bm-ebony overexpression induced a more yellowish coloration than $\mathrm{Bm}$ aaNAT in the black striped $p^{S}$ strain (Fig. 7). This is consistent with the previous observations that cuticles sclerotized exclusively by NADA (product of aaNAT) are colourless or very lightly straw-coloured, and grow darker brown the more NBAD (product of ebony) dominates in the process (Fig. 7) ${ }^{23}$. Furthermore, in contrast to the control silkworms whose markings were black throughout the larval stage, coloration differed between stages in Bm-aaNAT- and Bm-ebonyoverexpressing silkworms (Fig. 7). For example, $p^{S}$ larvae with ectopic Bm-aaNAT expression showed gold-brownish coloration in the third and fourth instars (Supplementary Figs S3 and 4 online), and greyish coloration in the fifth instar (Fig. 2). As both aaNAT and Ebony use dopamine as a substrate and do not affect the production of dopa-melanin, the differences in the coloration between larval stages may reflect the increase of grey dopamelanin in the elder instars (Fig. 7). In the control larvae, grey dopa-melanin may be concealed by black dopamine-melanin, leaving its presence undetectable.

In the Bm-ebony-overexpressing larvae, more significant differences in coloration between larval stages and areas were observed. It is noteworthy that only a subtle color change was observed in neonatal larvae and some areas remained black in the third-instar larvae with Bm-ebony overexpression (Figs 1, 3 and 7). As $\beta$-alanine is required for the conversion of dopamine to NBAD by Ebony (Fig. 7), a possible cause of these observations may be a difference in the uptake or accumulation of $\beta$-alanine. $\beta$-Alanine is produced by the action of the product of black gene (Fig. 7) in D. melanogaster and T. castaneum, whose adult black mutants are dark and black, respectively ${ }^{45}$. In the silkworm, a previous microarray analysis shows that the black gene is not expressed in the first instar ${ }^{46}$, suggesting that ectopic Bm-ebony expression could not alter neonatal larval coloration because of lack of $\beta$-alanine. The ubiquitous effect of aaNAT may be because the widely abundant acetyl-coA is its cofactor for the conversion of dopamine to NADA.

Until now, the 'visible markers' used for insect transgenesis were based on D. melanogaster mutants and mostly restricted to the same species, due to the lack of mutants to use as host strains 


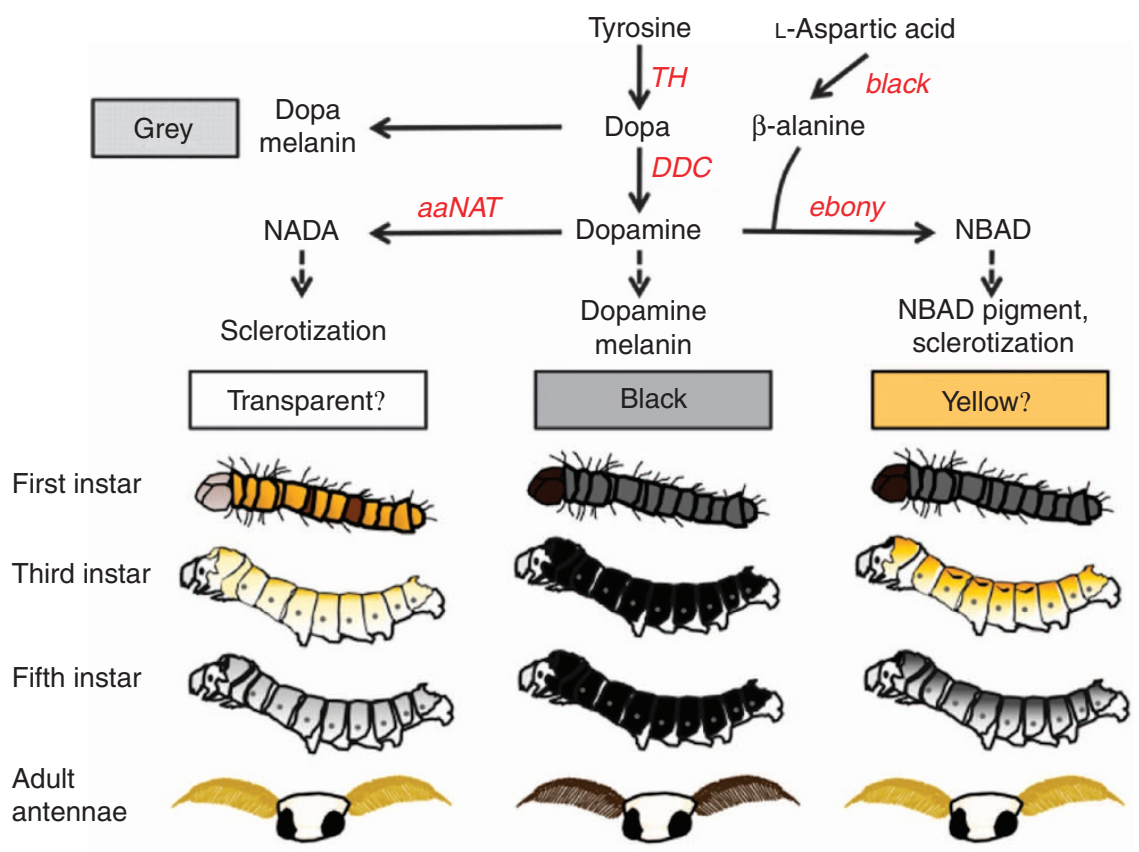

Figure 7 | Effects of overexpression of Bm-aaNAT and Bm-ebony on silkworm pigmentation. The striped strain $p^{S}$ is used in the model for larval coloration. The melanin synthesis pathway is adapted from Futahashi et al. ${ }^{16}$ The melanic pigmentation of silkworm larvae and adult antennae is affected by Bm-aaNAT and Bm-ebony, which use dopamine as substrate and produce transparent and yellowish cuticle, respectively. Notably, Bm-aaNAT effect on melanic pigmentation can be easily detected at the first instar, whereas Bm-ebony does not have significant effect in this stage. TH, tyrosine hydoxylase; DDC, dopa decarboxylase.

in other insects. As pigmentation is realized by the action of many genes, a dominant molecular marker that produces pigments where they did not originally exist seemed to be challenging. Our experiments indicate that a 'visible marker' based on melanin pigments can be developed using the opposite approach, that is, by reducing pigmentation. As this strategy led to visible changes in larvae of all stages and even in adults, the aaNAT transgene should enable the screening of transgenic insects during most of their lifetime (Fig. 7). The application of the aaNAT transformation marker should facilitate the use of transgenic techniques and promote molecular studies in insects.

\section{Methods}

Insects. The silkworm strains w1-pnd, actin A3-GAL4/3xP3-DsRed2 $(w 1)^{47}$ and C146 are maintained at the Transgenic Silkworm Research Unit of NIAS, and the $p^{S}$ strain at the Genetic Resource Center of NIAS. Silkworms were reared at $25^{\circ} \mathrm{C}$ with mulberry leaves or artificial diets (Nihon Nosan Kogyo, Yokohama, Japan) under a 16-h light: 8-h dark photoperiod. D. melanogaster was reared on a standard medium at $27^{\circ} \mathrm{C}$. The embryos used as recipients for DNA injection to generate transgenic lines were Oregon R. The GAL4 lines used in this study is as follows; hsp-GAL4 $\left.\left(w{ }^{*}\right] ; P\{w[+m C]=G A L 4-H s p 70 . P B\} 2 / C y O\right)$ and pannier-GAL4 (ref. 38) (y1 w1118; $P\{w+m W . h s=$ GawB $\}$ pnrMD237/TM3, $P\{w+m C=U A S$ y.C\}MC2,Ser1). H. axyridis was purchased from Agrisect Inc. (Japan) and maintained as described previously ${ }^{5,48}$. The insects were reared at $30^{\circ} \mathrm{C}$, and heat shock cycles were applied from second instar. One cycle consisted of heat shock at $40{ }^{\circ} \mathrm{C} 1 \mathrm{~h}$ followed by recovery at $30^{\circ} \mathrm{C}$ for $5 \mathrm{~h}$.

Donor plasmid construction. To construct the pBacUAS-NAT/3xP3EGFP plasmid, the Bm-aaNAT ORF was amplified from p50T pupal wing cDNA with the primers pBacMCS-UAS-BlnI-Kozak-BmNAT1-ORF-s (5'-GCCTAGTAGACCTA GAAAAATCAAAATGGCTGTTACAAGTACAAGAGGC-3') and pBacMCSUAS-BlnI-BmNAT1-ORF-a (5'-GTATGGCTGACCTAGTTACAGCTCCTTAAT GTAGACCCTG-3'), using Phusion Hot Start II High-Fidelity DNA Polymerase (New England Biolabs). The resulting PCR product was gel purified using a QIAquick Gel Extraction Kit (Qiagen) and subsequently cloned into the BlnI site of pBacUASMCS-3xP3EGFP using In-Fusion HD Cloning Kit (Clontech). The sequence was confirmed by the Sanger method using an ABI3130xl Genetic Analyser (Applied Biosystems). The Bm-aaNAT ORF sequence is the same as that with GenBank accession no. DQ256382.
To construct the pBacUAS-ebony/3xP3EGFP plasmid, the Bm-ebony ORF was amplified from p50T pupal wing cDNA with the primers pBacMCS-UAS-BlnIKozak-Bm-ebony-ORF-s (5'-GCCTAGTAGACCTAGAAAAATCAAAATGGGCT CCTTGCCTCGCGT-3') and pBacMCS-UAS-BlnI-Bm-ebony-ORF-a (5'-GTATG GCTGACCTAGTTAGTCCAAGGGTTTCCAGCAC- $3^{\prime}$ ). Subsequent procedures are the same as described above. The sequence of the Bm-ebony ORF was the same as that for GenBank accession no. AB455231.

To construct the pBacIE1-NAT/3xP3EGFP plasmid, the IE1 portion of pIEx-2 (Novagen) was amplified with the primers hr5-IE1-BglII-s (5'-CATTGCGTAGAT CTCGCGTAAAAC- $\left.3^{\prime}\right)$ and hr5-IE1-BlnI-a (5'-AAAAAAAACCTAGGTCACTTG GTTGTTCACGATC- $3^{\prime}$ ) and ligated into the 6-kb portion of BglII- and BlnIdigested pBacUASMCS-3xP3EGFP. The resulting plasmid was named pBac-IE13xP3EGFP. Next, the Bm-aaNAT ORF was amplified from pBacUAS-NAT/ 3xP3EGFP with the primers pBacIE1BlnIKozakBmNAT1ORF-s-2 (5'-AACCAAG TGACCTAGGAAAAATCAAAATGGCTGTTACAAGTACAAGAGGC- $3^{\prime}$ ) and pBacMCS-UAS-BlnI-BmNAT1-ORF-a (5'-GTATGGCTGACCTAGTTACAGCTC CTTAATGTAGACCCTG- $3^{\prime}$ ), and was subcloned into the $B \ln$ I site of pBac-IE1-3xP3EGFP using an In-Fusion HD Cloning Kit.

To construct the pBac-hsp-NAT/3xP3EGFP plasmid, the $h s p$ promoter portion was amplified with the primers Dmhsp70p-BglII-s (5'-AAAAAAAAA GATCTAAGCTTCTAGAATCCCAAAACAAACTGG-3') and Dmhsp70p-BlnI-a ( $5^{\prime}$-AAAAAAAACCTAGGGAATTCCCAATTCCCTATTCAGAG- $\left.3^{\prime}\right)$, and $p 10$ gene $3^{\prime}$ UTR (to enhance protein production ${ }^{49}$ ) portion was amplified with primers p10-5' -BlnI-s (5'-AAAAAAAACCTAGGATGAATCGTTTTTAAAATAACAAAT C-3') and p10-3'-PstI-a (5' -AAAAAACTGCAGGTTAACTCGAATCGCTATCCA AG- $3^{\prime}$ ), ligated into the $5.5-\mathrm{kb}$ portion of BglII- and PstI-digested pBacUASMCS$3 x$ PEGFP. The resulting plasmid was named pBac- $h s p-3 x$ P3EGFP. Next, the $B m-a a N A T$ ORF was amplified from $\mathrm{pBacUAS}-N A T / 3 \times \mathrm{X} 3 E G F P$ with the primers Syn21-NAT-s (5'-TTGGGAATTCCCTAGGAACTTAAAAAAAAAAATCAAAA TGGCTGTTACAAGTACAAGAGGC- $\left.{ }^{\prime}\right)$ and P10-NAT-a ( $5^{\prime}$-AACGATTCATC CTAGGTTACAGCTCCTTAATGTAGACCCTG- $3^{\prime}$ ), and was subcloned into the $B \ln \mathrm{I}$ site of pBac-hsp-3xP3EGFP using an In-Fusion HD Cloning Kit.

Generation of transgenic insects. Germline transformation of silkworms using the w1-pnd strain was conducted as described previously ${ }^{50}$. Transgenic silkworm generation using the C146 strain was conducted according to a previously described method ${ }^{51}$, with modifications. To prevent entering into diapause, eggs were treated with $20 \% \mathrm{HCl}$ for $30 \mathrm{~min}$ at $25^{\circ} \mathrm{C} 4 \mathrm{~h}$ after oviposition ${ }^{51}$. Donor and helper plasmid DNAs were injected into 476 eggs $30 \mathrm{~min}$ to $3 \mathrm{~h}$ after acid treatment. After injection, the embryos were incubated at $25^{\circ} \mathrm{C}$ to allow them to hatch. The hatch rate for HCl-treated C146 eggs was 26\% (125 out of 476) for injected eggs and $\sim 90 \%$ without injection. G1 screening was conducted by visual inspection on day 1 of first instar and was confirmed by fluorescence microscopy. 
Germline transformation of D. melanogaster was conducted as described previously ${ }^{52,53}$, using Oregon $\mathrm{R}$ as the host strain. PiggyBac-mediated germline transformation of ladybird beetle $H$. axyridis was conducted as described by Kuwayama et al..$^{5}$ Transgene screening was conducted by fluorescence microscopy at first instar.

Fitness assessment of IE1-NAT silkworms. For each experiment, non-transgenic siblings were used as control. Survival rate, percentage of eclosed adults compared with first-instar larvae; developmental time, amount of days it took from hatching to eclosion; fecundity, number of eggs laid per female within $24 \mathrm{~h}$ of separation from male; coccon weight, weight of coccon after removal of pupa.

Expression analysis of Bm-aaNAT in $\boldsymbol{H}$. axyridis. Total RNA was isolated from H. axyridis larva just before third moult using ISOGEN (Nippon gene), and reverse transcribed with a random primer (N6) using the first-strand cDNA synthesis kit (GE Healthcare) as previously described ${ }^{16}$. PCR conditions for Bm-aaNAT and Ha-rp49 (H. axyridis ribosomal protein 49 gene) were as follows: 35 cycles of $94{ }^{\circ} \mathrm{C}$ for $30 \mathrm{~s}, 55^{\circ} \mathrm{C}$ for $30 \mathrm{~s}$ and $72^{\circ} \mathrm{C}$ for $30 \mathrm{~s}$. Primers used for PCR are as follows; Ha-rp49 no. 1 (5'-GCGATCGCTATGGAAAACTC-3'), Ha-rp49 no. 2 $\left(5^{\prime}\right.$-TACGATTTTGCATCAACAGT- $\left.3^{\prime}\right)$, BmNAT1-F1 (5'-CTGCCGGAGACTT GTGACTC- $\left.3^{\prime}\right)$ and BmNAT1-R2 (5'-CATCTTGTAATTGAGCCTCTC- $3^{\prime}$ ).

\section{References}

1. Wimmer, E. A. Innovations: applications of insect transgenesis. Nat. Rev. Genet. 4, 225-232 (2003).

2. Robinson, A. S., Franz, G. \& Atkinson, P. W. Insect transgenesis and its potential role in agriculture and human health. Insect Biochem. Mol. Biol. 34, 113-120 (2004).

3. Atkinson, P. W. \& O’Brochta, D. A. In Encyclopedia of Insects. 2nd edn (eds Resh Vincent, H. \& Cardé Ring, T.) 410-416 (Academic Press, 2009).

4. Lorenzen, M. D. et al. piggyBac-mediated germline transformation in the beetle Tribolium castaneum. Insect Mol. Biol. 12, 433-440 (2003).

5. Kuwayama, H., Yaginuma, T., Yamashita, O. \& Niimi, T. Germ-line transformation and RNAi of the ladybird beetle, Harmonia axyridis. Insect Mol. Biol. 15, 507-512 (2006).

6. Sumitani, M., Yamamoto, D. S., Oishi, K., Lee, J. M. \& Hatakeyama, M. Germline transformation of the sawfly, Athalia rosae (Hymenoptera: Symphyta), mediated by a piggyBac-derived vector. Insect Biochem. Mol. Biol. 33, 449-458 (2003).

7. Nakamura, T. et al. Imaging of transgenic cricket embryos reveals cell movements consistent with a syncytial patterning mechanism. Curr. Biol. 20, 1641-1647 (2010).

8. Klemenz, R., Weber, U. \& Gehring, W. J. The white gene as a marker in a new P-element vector for gene transfer in Drosophila. Nucleic Acids Res. 15, 3947-3959 (1987).

9. Horn, C., Jaunich, B. \& Wimmer, E. A. Highly sensitive, fluorescent transformation marker for Drosophila transgenesis. Dev. Genes Evol. 210, 623-629 (2000).

10. Berghammer, A. J., Klingler, M. \& Wimmer, E. A. A universal marker for transgenic insects. Nature 402, 370-371 (1999).

11. Horn, C., Schmid, B. G., Pogoda, F. S. \& Wimmer, E. A. Fluorescent transformation markers for insect transgenesis. Insect Biochem. Mol. Biol. 32, 1221-1235 (2002).

12. Thomas, J. L., Da Rocha, M., Besse, A., Mauchamp, B. \& Chavancy, G. 3xP3-EGFP marker facilitates screening for transgenic silkworm Bombyx mori L. from the embryonic stage onwards. Insect Biochem. Mol. Biol. 32, 247-253 (2002).

13. Arakane, Y., Muthukrishnan, S., Beeman, R. W., Kanost, M. R. \& Kramer, K. J. Laccase 2 is the phenoloxidase gene required for beetle cuticle tanning. Proc. Natl Acad. Sci. USA 102, 11337-11342 (2005).

14. Futahashi, R., Banno, Y. \& Fujiwara, H. Caterpillar color patterns are determined by a two-phase melanin gene prepatterning process: new evidence from tan and laccase2. Evol. Dev. 12, 157-167 (2010).

15. Futahashi, R. \& Fujiwara, H. Melanin-synthesis enzymes coregulate stagespecific larval cuticular markings in the swallowtail butterfly, Papilio xuthus. Dev. Genes Evol. 215, 519-529 (2005).

16. Futahashi, R. et al. yellow and ebony are the responsible genes for the larval color mutants of the silkworm Bombyx mori. Genetics 180, 1995-2005 (2008).

17. Futahashi, R. et al. Laccase 2 is required for cuticular pigmentation in stinkbugs. Insect Biochem. Mol. Biol. 41, 191-196 (2011).

18. Wittkopp, P. J., True, J. R. \& Carroll, S. B. Reciprocal functions of the Drosophila yellow and ebony proteins in the development and evolution of pigment patterns. Development 129, 1849-1858 (2002).

19. Hiruma, K. \& Riddiford, L. M. The molecular mechanisms of cuticular melanization: the ecdysone cascade leading to dopa decarboxylase expression in Manduca sexta. Insect Biochem. Mol. Biol. 39, 245-253 (2009).

20. Hiruma, K., Riddiford, L. M., Hopkins, T. L. \& Morgan, T. D. Roles of dopa decarboxylase and phenoloxidase in the melanization of the tobacco hornworm and their control by 20-hydroxyecdysone. J. Comp. Physiol. B 155, 659-669 (1985).

21. Kramer, K. J. et al. Catecholamines and beta-alanine in the red flour beetle, Tribolium Castaneum-roles in cuticle sclerotization and melanization. Insect Biochem. 14, 293-298 (1984).

22. Andersen, S. O. Insect cuticular sclerotization: a review. Insect Biochem. Mol. Biol. 40, 166-178 (2010).

23. Andersen, S. O. In Comprehensive Molecular Insect Science. (eds Gilbert, I., Iatrou Kostas, L. \& Gill Sarjeet, S.) 145-170 (Elsevier, 2005).

24. Karlson, P. \& Sekeris, C. E. N-Acetyl-dopamine as screlotizing agent of the insect cuticle. Nature 195, 183-184 (1962).

25. Amherd, R., Hintermann, E., Walz, D., Affolter, M. \& Meyer, U. A. Purification, cloning, and characterization of a second arylalkylamine N-acetyltransferase from Drosophila melanogaster. DNA Cell Biol. 19, 697-705 (2000).

26. Hintermann, E., Grieder, N. C., Amherd, R., Brodbeck, D. \& Meyer, U. A. Cloning of an arylalkylamine $\mathrm{N}$-acetyltransferase (aaNAT1) from Drosophila melanogaster expressed in the nervous system and the gut. Proc. Natl Acad. Sci. USA 93, 12315-12320 (1996).

27. Hintermann, E., Jeno, P. \& Meyer, U. A. Isolation and characterization of an arylalkylamine $\mathrm{N}$-acetyltransferase from Drosophila melanogaster. FEBS Lett. 375, 148-150 (1995).

28. Tsugehara, T., Iwai, S., Fujiwara, Y., Mita, K. \& Takeda, M. Cloning and characterization of insect arylalkylamine $\mathrm{N}$-acetyltransferase from Bombyx mori. Comp. Biochem. Physiol. B Biochem. Mol. Biol. 147, 358-366 (2007).

29. Bembenek, J., Sakamoto, K. \& Takeda, M. Molecular cloning of a cDNA encoding arylalkylamine $\mathrm{N}$-acetyltransferase from the testicular system of Periplaneta americana: primary protein structure and expression analysis. Arch. Insect Biochem. Physiol. 59, 219-229 (2005).

30. Bembenek, J., Sehadova, H., Ichihara, N. \& Takeda, M. Day/night fluctuations in melatonin content, arylalkylamine $\mathrm{N}$-acetyltransferase activity and NAT mRNA expression in the CNS, peripheral tissues and hemolymph of the cockroach, Periplaneta americana. Comp. Biochem. Physiol. B Biochem. Mol. Biol. 140, 27-36 (2005).

31. Brodbeck, D. et al. Molecular and biochemical characterization of the aaNAT1 (Dat) locus in Drosophila melanogaster: differential expression of two gene products. DNA Cell Biol. 17, 621-633 (1998).

32. Ichihara, N., Okada, M. \& Takeda, M. Characterization and purification of polymorphic arylalkylamine $\mathrm{N}$-acetyltransferase from the American cockroach, Periplaneta americana. Insect Biochem. Mol. Biol. 32, 15-22 (2001).

33. Mehere, P., Han, Q., Christensen, B. M. \& Li, J. Identification and characterization of two arylalkylamine $\mathrm{N}$-acetyltransferases in the yellow fever mosquito, Aedes aegypti. Insect Biochem. Mol. Biol. 41, 707-714 (2011).

34. Dai, F. Y. et al. Mutations of an arylalkylamine-N-acetyltransferase, BmiAANAT, are responsible for silkworm melanism mutant. J. Biol. Chem. 285, 19553-19560 (2010).

35. Zhan, S. et al. Disruption of an N-acetyltransferase gene in the silkworm reveals a novel role in pigmentation. Development 137, 4083-4090 (2010).

36. Sakudoh, T. et al. Carotenoid silk coloration is controlled by a carotenoidbinding protein, a product of the Yellow blood gene. Proc. Natl Acad. Sci. USA 104, 8941-8946 (2007).

37. Tamura, T., Kuwabara, N., Uchino, K., Kobayashi, I. \& Kanda, T. An improved DNA injection method for silkworm eggs drastically increases the efficiency of producing transgenic silkworms. J. Insect Biotechnol. Sericol. 76, 155-159 (2007).

38. Calleja, M., Moreno, E., Pelaz, S. \& Morata, G. Visualization of gene expression in living adult Drosophila. Science 274, 252-255 (1996).

39. Teule, F. et al. Silkworms transformed with chimeric silkworm/spider silk genes spin composite silk fibers with improved mechanical properties. Proc. Natl Acad. Sci. USA 109, 923-928 (2012).

40. Hines, H. M. et al. Transcriptome analysis reveals novel patterning and pigmentation genes underlying Heliconius butterfly wing pattern variation. BMC Genomics 13, 288 (2012).

41. Rebeiz, M., Pool, J. E., Kassner, V. A., Aquadro, C. F. \& Carroll, S. B. Stepwise modification of a modular enhancer underlies adaptation in a Drosophila population. Science 326, 1663-1667 (2009).

42. Shirataki, H., Futahashi, R. \& Fujiwara, H. Species-specific coordinated gene expression and trans-regulation of larval color pattern in three swallowtail butterflies. Evol. Dev. 12, 305-314 (2010).

43. Koch, P. B., Behnecke, B. \& ffrench-Constant, R. H. The molecular basis of melanism and mimicry in a swallowtail butterfly. Curr. Biol. 10, 591-594 (2000).

44. Ferguson, L. C., Maroja, L. \& Jiggins, C. D. Convergent, modular expression of ebony and tan in the mimetic wing patterns of Heliconius butterflies. Dev. Genes. Evol. 221, 297-308 (2011). 
45. Arakane, Y. et al. Molecular and functional analyses of amino acid decarboxylases involved in cuticle tanning in Tribolium castaneum. J. Biol. Chem. 284, 16584-16594 (2009).

46. Liang, J., Zhang, L., Xiang, Z. \& He, N. Expression profile of cuticular genes of silkworm, Bombyx mori. BMC Genomics 11, 173 (2010).

47. Imamura, M. et al. Targeted gene expression using the GAL4/UAS system in the silkworm Bombyx mori. Genetics 165, 1329-1340 (2003).

48. Niimi, T., Kuwayama, H. \& Yaginuma, T. Larval RNAi applied to the analysis of postembryonic development in the ladybird beetle, Harmonia axyridis. J. Insect Biotechnol. Sericol. 74, 95-102 (2006).

49. Pfeiffer, B. D., Truman, J. W. \& Rubin, G. M. Using translational enhancers to increase transgene expression in Drosophila. Proc. Natl Acad. Sci. USA 109, 6626-6631 (2012).

50. Tamura, T. et al. Germline transformation of the silkworm Bombyx mori L. using a piggyBac transposon-derived vector. Nat. Biotechnol. 18, 81-84 (2000).

51. Inoue, S. et al. A fibroin secretion-deficient silkworm mutants $\mathrm{Nd}-\mathrm{s}(\mathrm{D})$, provides an efficient system for producing recombinant proteins. Insect Biochem. Mol. 35, 51-59 (2005).

52. Rubin, G. M. \& Spradling, A. C. Genetic transformation of Drosophila with transposable element vectors. Science 218, 348-353 (1982).

53. Hara, K., Kuwayama, H., Yaginuma, T. \& Niimi, T. Establishment of a tetracycline-off system using a piggyBac-based vector as a gene functional analysis tool for the temporal targeting of gene expression. J. Insect Biotechnol. Sericol. 77, 159-166 (2008).

\section{Acknowledgements}

We thank Marian Goldsmith for the critical reading of the manuscript and valuable advice. We are obliged to Masatsugu Hatakeyama and Shuichiro Tomita for technical assistance. We are grateful to Kazuei Mita for the sequencing of the Bm-aaNAT and $B m$-ebony cDNA clones, to Tetsuya Iizuka for the $p^{S}$ and C146 strains, arearing of G0 transgenic silkworms and cocoon yield information, to Koji Hashimoto and Kaoru Nakamura for technical support with silkworm germline transformation, and to Haruka Kawaguchi for care of ladybird beetles. This work was supported by grants from the Ministry of Agriculture, Forestry and Fisheries of Japan.

\section{Author contributions}

M.O., R.F., T.T. and H.S. designed research; M.O., K.U., J. H., T. O. and T.N., performed research; M.O. and T. N. analysed the data; M.O. wrote the paper with the help of R.F., T.T., T.N. and H.S.

\section{Additional information}

Supplementary Information accompanies this paper at http://www.nature.com/ naturecommunications

Competing financial interests: The authors declare no competing financial interests.

Reprints and permission information is available online at http://npg.nature.com/ reprintsandpermissions/

How to cite this article: Osanai-Futahashi, M. et al. A visible dominant marker for insect transgenesis. Nat. Commun. 3:1295 doi: 10.1038/ncomms2312 (2012).

(c) (1) (2) This work is licensed under a Creative Commons AttributionCon NC $_{\text {SA }}$ Nommercial-ShareAlike 3.0 Unported License. To view a copy of this license, visit http://creativecommons.org/licenses/by-nc-sa/3.0/ 